\title{
Coupling Time-Resolved in situ Experiments with Three-Dimensional Imaging - a New Approach to Defect Evolution Studies
}

\author{
G.S. Liu, ${ }^{*}$ J. Kacher* and I.M. Robertson* \\ *Dept. of Materials Science and Engineering, University of Illinois, Urbana, IL 61801.
}

Diffraction contrast imaging coupled with the $\vec{g} \cdot \vec{R}$ invisibility condition has been used extensively to characterize the microstructure at a specific instance in time. Capturing the complete microstructural evolution, however, requires examining samples extracted from many interrupted tests, assuming each to be representative of that point in the process, and conducting a posteriori analysis to suggest how the microstructure proceeds from one state to the next. Knowledge of the pertinent processes and mechanisms is accrued by using the microscope as an experimental laboratory in which the experiments and reactions are conducted and observed in real time. In addition, the spatial component of the microstructure can be recovered by using electron tomography reconstruction techniques. Here we propose to combine both approaches such that the evolution is followed in an experiment conducted in the microscope with images for reconstructing a three-dimensional tomogram or model acquired periodically.

Combining both approaches depends on several breakthroughs, some of which will be achieved through instrumentation and others through advances in reconstruction algorithms. In the case of electron tomography for defect analysis, it will also be necessary to overcome several challenges: Reducing the angular range, typically $\pm 70^{\circ}$, and the number of images acquired, typically every 1 degree; relaxing uniform contrast requirements across a micrograph and across all acquired images; and minimizing the time needed to acquire the image set. Some of these constraints can be alleviated by using the tomogram to produce a structural model in which prior knowledge of the dislocations is used or by using fiducial markers to construct the model itself. Additionally, dual-axes tomography approaches with non-orthogonal diffraction conditions are being used to ensure all dislocations are captured in the tomogram and model, and a strategy for incorporating the real space coordinate system has been developed. The last feature is essential for analyzing defect structures, especially dislocations and slip system determination.

The challenge of acquiring an image every degree over a large angular range can be mitigated if knowledge about dislocations is used to generate the model. This is feasible because the reconstruction need only possess sufficient resolution for a three-dimensional model to be constructed. An example of this is shown in Figure 1, in which the three-dimensional dislocation structure around an $\mathrm{Al}_{3} \mathrm{Sc}$ precipitate in an Al matrix from the tomogram and the model are compared. Also shown is the Thompson tetrahedron, which defines the coordinate system within the tomogram and model [1]. For less complex dislocation structures, it is possible to remove the constraint of maintaining the same diffraction condition over the angular range and at the same time reduce the number of images needed to enable the reconstruction. Here fiducial markers are positioned at the location at which the dislocation intersects the free surfaces, this is done for images acquired at different angles about a known tilt direction. For the reconstruction, the TEM image is removed and the markers alone are used to generate the three-dimensional structure. This approach, which is valid for straight dislocations that run directly between the two specimen surfaces, uses just ten images from which to reconstruct a usable model. An example comparing the image quality in the tomogram and model generated using just ten 
images is shown in Figure 2 for dislocations interacting with a $\Sigma=3$ boundary in 304 stainless steel. This development brings us closer to the point at which periodic snapshots of an evolving structure can be attained during a deformation experiment [2].

\section{References}

[1] G.S. Liu, I.M. Robertson. J. Mater. Res. 26 (2011).

[2] J.P. Kacher et al. Scripta Mater.64 (2011) 677-680.

[3] This work was supported by the US Department of Energy, Office of Basic Energy Sciences under contract DE-FG02-07ER46443.
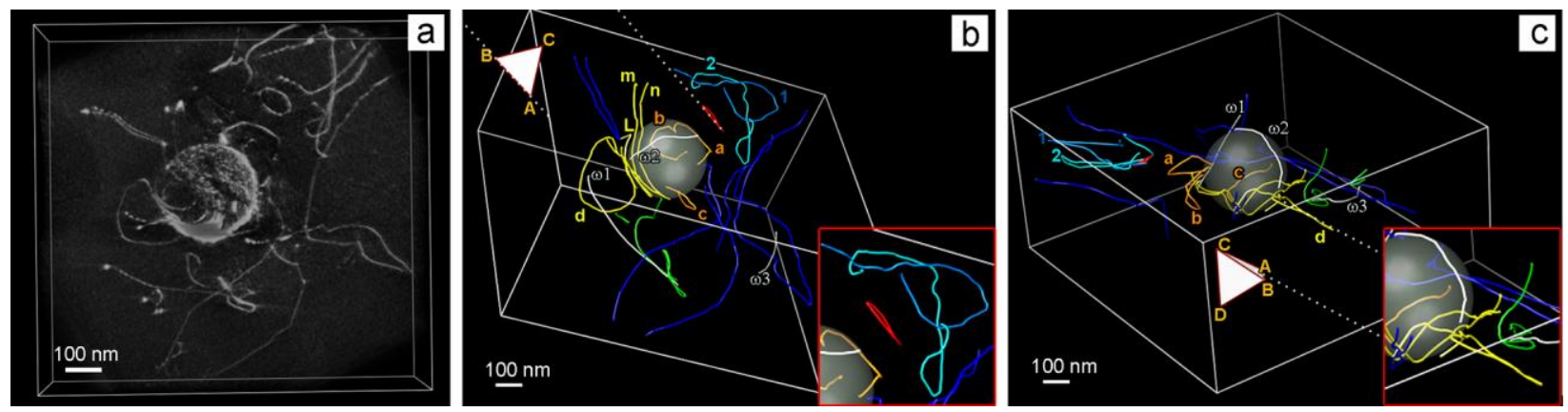

Figure 1. An $\mathrm{Al}_{3} \mathrm{Sc}$ particle surrounded by a complex arrangement of matrix dislocations. (a) reconstructed tomogram produced from a tilt series acquired with $\mathbf{g}=200$. (b-c) threedimensional traced model of the same particle from two views, with insets showing details of the interaction. Crystallographic directions and slip planes are indicated by an embedded Thompson tetrahedron [1].
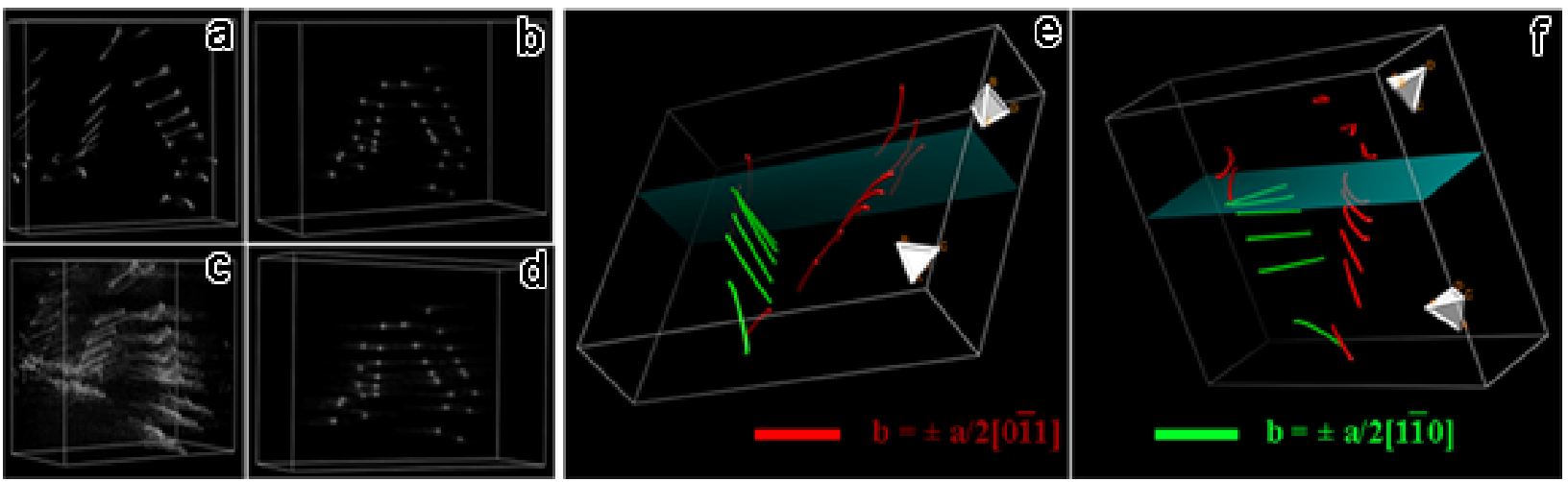

Figure 2. Dislocations in 304 SS impinging on a $\Sigma 3$ boundary. Tomogram of the area of interest reconstructed (a) from 40 TEM images and (b) from 40 fiducial images; no significant difference is seen. Tomograms reconstructed from (c) 10 TEM images and (d) 10 fiducial images; the tomogram constructed with the fiducial method retains fidelity. (e-f) three-dimensional traced model produced from the tomogram in Fig.2(d), from two different views [2]. 